
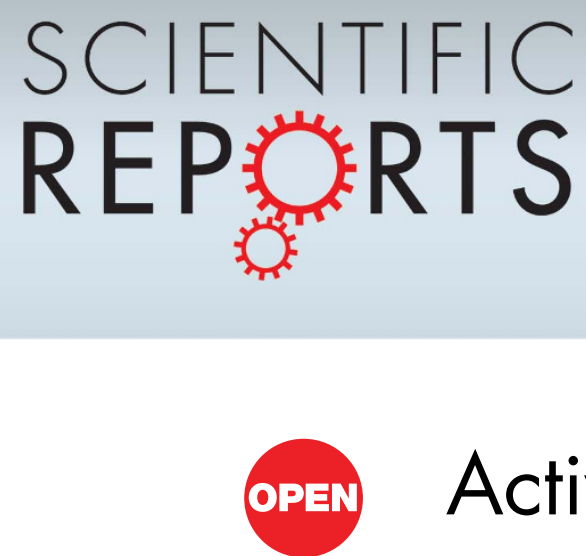

\title{
Activating optomechanical entanglement
}

\author{
Laura Mazzola ${ }^{1,2} \&$ Mauro Paternostro²
}

SUBJECT AREAS:

QUANTUM PHYSICS

QUANTUM OPTICS

THEORETICAL PHYSICS

INFORMATION THEORY AND COMPUTATION

Received

22 November 2011

Accepted

30 November 2011

Published

20 December 2011

Correspondence and requests for materials should be addressed to L.M. (I.mazzola@qub. ac.uk)

\begin{abstract}
${ }^{1}$ Turku Centre for Quantum Physics, Department of Physics and Astronomy, University of Turku, Fl-20014 Turun yliopisto, Finland, ${ }^{2}$ Centre for Theoretical Atomic, Molecular and Optical Physics, School of Mathematics and Physics, Queen's University Belfast, BT7 INN Belfast, United Kingdom.
\end{abstract}

We propose an optomechanical setup where the activation of entanglement through the pre-availability of non-classical correlations can be demonstrated experimentally. We analyse the conditions under which the scheme is successful and relate them to the current experimental state of the art. The successful activation of entanglement embodies an interesting alternative to current settings for the revelation of fully mechanical nonclassicality.

U Nderstanding quantum correlations and their role is one of the key goals of the current research in modern quantum physics ${ }^{1}$. Although much of the attention has so far been focused on entaglement as the form of quantum correlations to look at when dealing, for instance, with quantum computational speed-up and better-than-classical communication capabilities, such a perspective has been challenged by the identification of other forms of non-classical correlations going beyond entanglement ${ }^{2-7}$. Needless to say, as entanglement is the unique form of quantum correlations for pure states, the arena were the role (if any) that such quantum correlation should be investigated is the one embodied by mixed quantum states. For such states, entangled is no longer synonymous of non-classical and other quantifiers have been proposed, each striving to grasp specific aspect through which quantumness of correlations manifests itself. Among them, quantum discord ${ }^{2}$ has so far enjoyed a growing popularity, notwithstanding the difficulties inherent in its analytic formulation even for simple two-qubit states, due to the intriguing implications it is alleged to have for the speed-up of some protocols for quantum computing ${ }^{8}$ and its operational interpretation ${ }^{9,10}$.

Remarkably, it has been very recently realized that an effective way to understand discord and its role in quantum information processing could be the establishment of tight relations with entanglement itself. In particular, Refs. ${ }^{11,12}$ have shown that, in the $n$-qudit scenario aided by unitary quantum gates, discord might be interpreted as the resource whose availability enables physically relevant effects, such as the production of an entangled state useful for quantum information processing, otherwise prevented when only classical correlations are at hand. Besides providing an alternative definition of classically correlated states, these results give a direct link between broader quantum correlations and the resource for quantum information processing. To date, although discord has been successfully extended to the domain of Gaussian continuous variable states ${ }^{13,14}$, it is not known whether a similar schemes hold in the continuous variable arena as well.

Here we provide strong evidence that, indeed, an entanglement activation protocol based on the preavailability of discord can be formulated for registers and ancillae having continuous spectra. In order to do so, we adapt the original formulation of Ref. ${ }^{12}$ to a realistic system of cavity quantum optomechanics that includes, $a b$ initio, environmental losses and decoherence, thus extending the scheme itself from the unitary to the opensystem domain. We use two independent optomechanical cavities ${ }^{15-17}$ : the register is embodied by the mechanical mirrors, the ancillae by the cavity fields. We show that pre-avaliable quantum correlations in the state of the mechanical subsystem can always be transformed into entanglement involving the mechanical and the optical systems. Besides its fundamental relevance, our study can be seen from the perspective of effective diagnostics on the purely mechanical state: the current experimental progresses are such that optomechanical entanglement will soon be within reach thus proving the potential for experimental quantum information science held by the optomechanical arena ${ }^{18-21}$. By revealing the appearance of optomechanical entanglement, our scheme will give us indirect evidence of (much harder to access) nonclassicality at the mechanical level. Therefore, this study contributes to the grasping for a full comprehension of the interplay and relation between discord and entanglement and proves the possibility of activating the latter through the former in an experimentally realistic CV context. Furthermore, it opens up the way to further uses of optomechanical systems as nodes in quantum networks.

\section{Results}

Entanglement activation scheme. For clarity, here we briefly summarise the working principle of the entanglement activation scheme put forward in Ref. ${ }^{12}$ and illustrated in Fig. 1 (a). The protocol is performed 
(a)

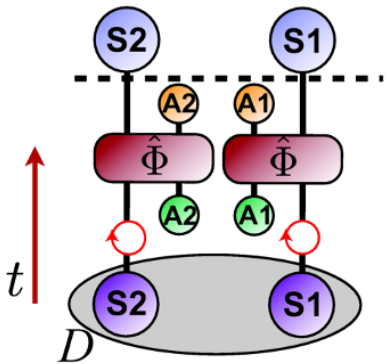

(b)

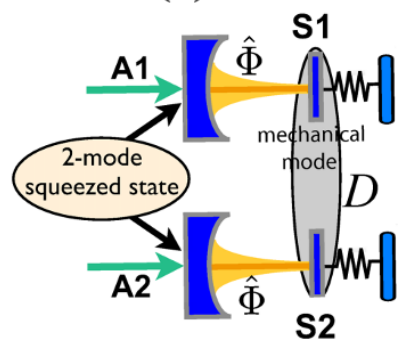

Figure 1 Scheme of principle of the activation protocol. (a) The register composed of systems $S_{j}(j=1,2)$ is initialized in a separable state and subjected to a set of local rotations (represented by the circled arrows). System $S_{j}$ then evolves jointly with the ancilla $A_{j}$ according to the completely positive map $\hat{\Phi}$. If the initial state of the register has non-zero discord, the output state after the application of the maps is entangled with respect to the system-ancilla bipartition $\left(S_{1} S_{2}\right)\left(A_{1} A_{2}\right)$. The arrow of time is also shown, while $D$ stands for the presence of discord. (b) Each system-ancilla unit is embodied by an optomechanical system consisting of an open optical resonator with a light, movable end mirror, pumped by an intense light field. The field-mirror radiation-pressure coupling, together with the lossy mechanisms affecting the device (see body of the manuscript for details) realize the map $\hat{\Phi}$. Separable yet discorded initial mechanical states are prepared by driving the cavities with a two-mode squeezed vacuum of

between two registers, the system register $\mathrm{S}$ (composed by $S_{j}$ with $j=$ $1, \ldots, n)$ and the ancilla register $\mathrm{A}$ (with elements $A_{j}$ ). The two registers are initialised in the factorized state $\rho_{S} \otimes \rho_{A}$, where $\rho_{S}$ is a separable state of the system register and $\rho_{A}=\otimes_{i=1}^{n} \rho_{A}^{i}$ is the tensor product of $n$ identical states of the ancilla register. A demon applies local random unitaries $\hat{U}_{i}$ on each $S_{j}$, which then undergo a joint evolution with the homonymous element of the ancillary register $A_{j}$. In general, such evolution is described by a completely positive dynamical map $\hat{\Phi}$. The key statement of reference ${ }^{12}$ is that iff $\rho_{S}$ is classically correlated (i.e., having zero discord) it is always possible for the demon to find a configuration $\hat{U}_{d}$ of demon's unitaries such that $\otimes_{i=1}^{n} \hat{\Phi}_{i}\left[\hat{U}_{d}\left(\rho_{S} \otimes \rho_{A}\right) \hat{U}_{d}^{\dagger}\right]$ is separable across the system-ancilla split. The result has been found under the assumptions of $i$ ) registers composed of qubits, $i$ ) a rigid set of unitary gates applied to the $S_{j}-A_{j}$ systems and $\mathrm{iii}$ ) ancillae initially prepared in a fiducial reference state.

Activation scheme in a realistic optomechanical device. We turn the key claim in Ref. ${ }^{12}$ somewhat upside-down and provide the following

Statement: Consider a register composed of $n$ non-interacting optomechanical systems, each interacting with a CV ancilla prepared in a fiducial initial state. If the elements of the register initially share nonclassical correlations, dynamical entanglement in the register-ancillae split can be created robustly against adversary effects of a demon that acts locally on the state of each element of the register itself.

As anticipated, the physical system comprises two optical resonators, each endowed with a mechanical end-mirror and interacting via radiation pressure with two optical modes ${ }^{15-17}$. Each cavity is pumped by an external laser field that is quasi-resonant with the cavity frequency $\omega_{c}^{i}$. The Hamiltonian of each optomechanical device, in a frame rotating at the frequency $\omega_{\mathrm{L}}$ of the external lasers (assumed to be the same), reads

$$
\hat{H}_{i}=\hbar \delta^{i} \hat{n}_{i}-\hbar \chi_{i} \hat{n}_{i} \hat{q}_{i}+\left(\frac{\hat{p}_{i}^{2}}{2 m_{i}}+\frac{m_{i} \omega_{m}^{i 2}}{2} \hat{q}_{i}^{2}\right)+i \hbar \varepsilon_{i}\left(\hat{c}_{i}^{\dagger}-\hat{c}_{i}\right),
$$

where $\hat{q}_{i}\left(\hat{p}_{i}\right)$ is the position (momentum) quadrature of the $\mathrm{i}^{\text {th }}$ mechanical system, $\hat{c}_{i}\left(\hat{c}_{i}^{\dagger}\right)$ is the annihilation (creation) operator of the $\mathrm{i}^{\text {th }}$

cavity field (whose photon-number operator and energy decay rate are $\hat{n}_{i}$ and $\mathrm{k}_{\mathrm{i}}$, respectively), and $\delta^{i}=\omega_{C}^{i}-\omega_{L}$ is the pump-cavity detuning. Each cavity has length $\mathrm{L}_{\mathrm{i}}$, so that $\chi_{i}=\omega_{C}^{i} / L_{i}$ is the associated cavity-mirror radiation pressure coupling rate. The frequency and mass of the $\mathrm{i}^{\text {th }}$ mechanical oscillator are $\omega_{m}^{i}$ and $\mathrm{m}_{i}$. Finally, by calling $\mathcal{P}_{1,2}$ the pumping power of each driving laser, we have $\varepsilon_{i}=\sqrt{2 \kappa_{i} \mathcal{P}_{i} /\left(\hbar \omega_{L}^{i}\right)}$.

The mechanical system is affected by decoherence induced by the thermal Brownian motion of the mechanical oscillators, which are damped at a rate $\gamma_{m}^{i}$. Together with the cavity losses that have already been mentioned, this makes our system explicitly open and the dynamics undertaken by the joint optomechanical device properly described in terms of the completely positive map $\rho_{\text {out }}=\otimes_{i=1}^{2} \hat{\Phi}_{i}\left[\rho_{\text {in }}\right]$ with $\rho_{\text {in }}$ the initial state of the joint mirror-light system. We provide details on the explicit form of each $\hat{\Phi}_{i}$ in the Methods section.

Activating entanglement from quantum discord. We now show how quantumness of correlations between the mirrors guarantees the generation of dynamical entanglement in the mirrors-light bipartition. In the Methods section we recall the definitions of discord, quantifying quantumness of correlation, and entanglement for a two-mode Gaussian CV states. Before discussing the actual results, we make a preliminary analysis of the entanglement created in a single optomechanical cavity between the mirror and the cavity mode as a function of the temperature of the Brownian bath the mirror is interacting with.

Radiation pressure is responsible for an in principle entangling dynamics between the mechanical mode and the cavity field ${ }^{22-28}$. However, such entanglement is the result of a delicate trade-off between the strength of the optomechanical coupling, the mechanical mass, the mechanical/optical quality factor and, quite crucially, temperature. The generation of entanglement during the transient evolution does not guarantee its persistence at the steady state achieved by taking the limit for $t \rightarrow \infty$ in Eq. (4). In particular, while the steady-state entanglement will depend only on the relative ratios of the critical parameters identified above, the dynamical one will have a strong dependence on the initialization of the mechanical register. This observation is key to the understanding of the working principles of our proposal.

In order to shed light on this point, we study a single optomechanical system and investigate the dynamical entanglement generated when the mechanical (optical) mode is prepared in a thermal (coherent) state. We consider the set of parameters listed in Table I, which are taken from a very recent experiment and represent the current experimental state of the $\operatorname{art}^{20}$ and determine the corresponding logarithmic negativity. In Fig. 2 (a) we show the maximum dynamical entanglement achieved within the time-window that precedes the reaching of the steady-state conditions corresponding to the choice of parameters mentioned above. We clearly see the existence of two distinct regions: in the low temperature region $(T<0.02 \mathrm{~K})$, there is always an instant of time at which dynamical entanglement is

Table I| Parameters used for the simulations run throughout the manuscript (we assume the same values for each optomechanical subsystem). Values taken from Ref. ${ }^{20}$

\begin{tabular}{lcc} 
Parameter & Symbol & Value \\
\hline Mechanical mass & $m$ & $145 \mathrm{ng}$ \\
Mechanical frequency & $\omega_{m}$ & $947 \mathrm{KHz}$ \\
Cavity length & $L$ & $25 \mathrm{~mm}$ \\
Input power & $\mathcal{P}$ & $11 \mathrm{~mW}$ \\
Cavity-field wavelength & $\lambda$ & $1064 \mathrm{~nm}$ \\
Optical damping rate & $\kappa / 2 \pi$ & $215 \mathrm{KHz}$ \\
Mechanical damping rate & $\gamma / 2 \pi$ & $140 \mathrm{~Hz}$ \\
\hline
\end{tabular}


(a)

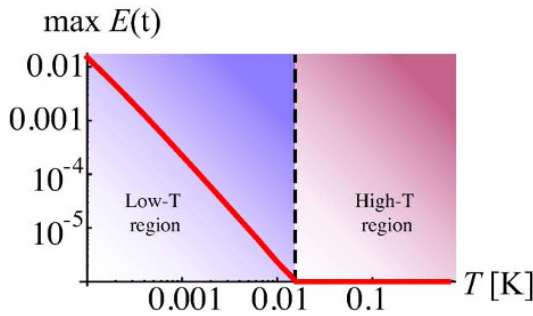

(b)

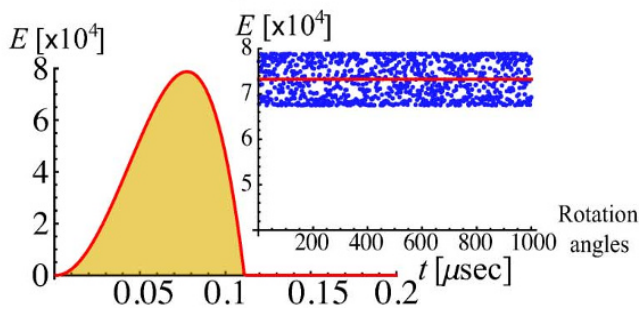

Figure 2 High-temperature entanglement activation. (a) The maximum value of dynamical entanglement created over time between the mechanical and optical mode as a function of the temperature of the brownian bath. (b) Optomechanical entanglement created between the field of one cavity and the corresponding mechanical mirror as a function of the interaction time. The entanglement in the other optomechanical subsystem shows an identical behavior. The initial state is the tensor product of coherent states of the cavity fields and a separable yet discorded state of the mirrors obtained using the

created. In the high temperature region where $T>0.02 \mathrm{~K}$, dynamical entanglement never arises. In our discussion on discord-enabled entanglement activation, we thus keep such two cases distinct.

Case 1): High temperature activation.- We now consider the case of two independent optomechanical subsystems, whose mechanical modes are assumed to be prepared in a thermal state at $T>0.02 \mathrm{~K}$ each. In this regime of parameters, the radiation pressure interaction is not dynamically entangling and, given the bilocal nature of the overall map, the entanglement in the mechanical-vs-optical bipartition has necessarily to be zero. We now show how this scenario changes when initial discord is considered.

Let us take the mechanical modes as prepared in a thermal state at $T=0.4 \mathrm{~K}$ each, therefore well in the region of no dynamical entanglement. Notice that this is also the temperature of a cryostat. Using the protocol put forward in Ref. ${ }^{29}$, which is based on the driving of two non-interacting optomechanical cavities with an entangled twomode squeezed state of light, we can create a separable yet nonclassically correlated state of the mechanical modes. For instance, using a two-mode drive having squeezing parameter $r=1$, the joint optomechanical device is such that $E=0$ with $D=0.002$. We now turn off the driving fields and shine the high-intensity uncorrelated pumps on each cavity (same parameters as for Fig. 2 (a) but for the temperature) so as to check for the possibility to activate entanglement. In Fig. 2 (b) we show how such initial fully mechanical nonclassical correlations allow to activate optomechanical entanglement in the mirrors-vs-cavity fields bipartition over a time window of the order of the decoherence time induced by the Brownian reservoirs. Needless to say, to be faithful to the scheme in Ref. ${ }^{12}$, we should incorporate the effects of the demon's actions over the register, i.e. the local rotations that are supposed to scramble the initial state of the register so as to prevent the activation of entanglement. Formally, this is equivalent to the application of the operator $\otimes_{i=1}^{2} e^{i \vartheta_{i}(t)\left(\hat{q}_{i}^{2}+\hat{p}_{i}^{2}\right)}$ to the state of the mechanical oscillators, i.e. the use of the covariance matrix $v_{\mathrm{C}}=R v R^{T}$ as the initial conditions of the dynamical map at the core of our investigation (here $\mathrm{R}=$ $\oplus_{i=1}^{2}\left[\begin{array}{ll}\cos \vartheta_{i} & \sin \vartheta_{i} \\ -\sin \vartheta_{i} & \cos \vartheta_{i}\end{array}\right]$ is the symplectic transformation associated with a phase-space rotation by the angles $\vartheta_{i}$ ). Experimentally, this is obtained by mismatching the time at which the mechanical modes start interacting with the respective pumping field: in the ideal unitary case (where all loss mechanisms are neglected) this would result in free dynamics of the mechanical modes for mutually unequal times. We have run our simulation by using a locally rotated initial mechanical state and finding that for no value of $\vartheta_{i}$ the entanglement in the output quadripartite optomechanical state disappears. This is illustrated in the inset of Fig. 2 (b), where we show the output entanglement for a sample of $10^{3}$ randomly rotated initial states. We are thus in a position to claim the validity of our Statement for Case 1) of our study.
A remark on the success of the protocol is due. Through the scheme described in $\operatorname{Ref}_{.}{ }^{29}$ and used here to initialize the mechanical register, we set quantum correlations in the state of the mechanical oscillators and, at the same time, cool them down to effective temperatures lower than that of their Brownian environment. In fact, the mean occupation number of the mechanical modes prepared in the separable but discorded state used here is as small as 12, against the 8437 thermal quanta that correspond to the initial mechanical state at $T=0.4 \mathrm{~K}$. In order to make sure that the activation of entanglement is guaranteed only by the availability of initial discord and not to the effectively cold system we are dealing with, we take a discorded state $\rho_{D}$ of the mirrors "as warm" as the thermal state at the Brownian bath, i.e. a mechanical state whose single-mode reductions have variances of the associated quadratures comparable in magnitude to those of a bipartite thermal state at $T=0.4 \mathrm{~K}$. Formally, this leaves reduced single-mode states that are classically squeezed: the associated covariance matrices are fully diagonal with only slightly unbalanced non-zero elements which are, nevertheless, larger than the vacuum limit 0.5 . From $\rho_{D}$ we build up a classically correlated bipartite state $\rho_{C C}$ obtained by taking the tensor product of such single-mode reductions (this is equivalent to discarding all the correlations in $v$ ). We then run the activation protocol using the covariance matrices $v_{D}$ and $v_{C C}$ associated with such states finding that only in the former instance the mirrors-vs-optical modes bipartition becomes dynamically entangled regardless of the correlationsscrambling local rotations, therefore reinforcing the central role played by discord.

Case 2): Low temperature activation.- For $T<0.02 \mathrm{~K}$, we know from Fig. 2 (a) that the optomechanical interaction sets dynamical entanglement within each optomechanical subsystem. The monogamy arguments used above ensure that also the mirrors-vs-cavity modes bipartition is dynamically entangled. This makes the assessment of entanglement activation under conditions of low temperature less interesting. Nevertheless, there is still room and significance for our activation procedure.

In fact, the local dynamical entanglement revealed in Fig. 2 (a) can be fully inhibited when considering, as initial states of the optical modes in the activation protocol, two single-mode squeezed states. Fig. 3 (a) shows the optomechanical entanglement created as a function of time and the local squeezing parameter for $T=0.03 \mathrm{mK}$. We notice that for high enough values of the local squeezing, the dynamical optomechanical entanglement is completely destroyed. Yet, fully in line with the high-temperature case, the presence of discord in the mechanical state leads to dynamical entanglement in the mirrors-vscavity fields bipartition. To demonstrate this we prepare first the mirrors in a discorded state by using the same protocol as before ${ }^{29}$ (again, we use a two-mode driving field with $r=1$, although any other choice gives qualitatively similar results), run the activation protocol and contrast the results with what is achieved by means of an initial mechanical state obtained from the separable discorded 
(a)

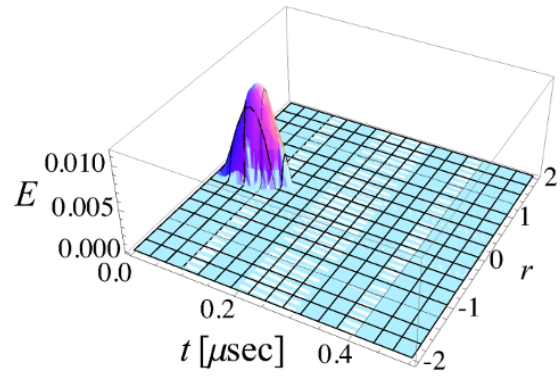

(b)

Figure 3 Low-temperature entanglement activation. (a) Optomechanical entanglement created between a single mechanical mode and its corresponding cavity field. Each mirror is prepared in a thermal state at the same temperature of its Brownian bath, while the optical mode is in a squeezed state. The optomechanical entanglement is shown against time and the squeezing parameter of the optical modes. (b) Optomechanical entanglement created in the two mirrors two light modes partition in the following initial conditions: light modes prepared in a single-mode squeezed state with squeezing parameter $r=0.5$,

one by deleting all the correlations (as before, this delivers a tensor product of classical squeezed mechanical states). Fig. 3 (b) shows the output entanglement created in the mirrors-light modes bipartition using these initial states. The solid (blue) line is for the entanglement generated using the discorded state, while the dashed (red) one embodies the entanglement generated from the factorized state of the mechanical modes. Clearly, the availability of quantum discord activates dynamical entanglement in quite a spectacular way. Such entanglement cannot be inhibited by means of local scrambling rotations by the demon, in a way fully in line with the analysis performed previously in the high-temperature case. In the long-time limit, finally, the two dynamical conditions converges towards the settlement of steady-state entanglement. The validity of the discord-based activation scheme remains thus vindicated.

\section{Discussion}

We have shown the existence of experimentally accessible conditions under which an entanglement activation protocol requiring the preavailability of quantum correlations in the form of discord holds in the CV scenario as well. While this extends significantly the breath of applicability of the framework nicely put forward in Ref. ${ }^{12}$ for the discrete-variable arena and using a rigid unitary gate between the register and the ancillae, we stress that our simulations have been run using the parameters used in very recent experiments on optomechanics. This dresses our analysis of further relevance as it embodies a proposal for the demonstration of interesting fundamental effects in a mature, realistic and extremely timely experimental setting.

\section{Methods}

Optomechanical evolution. Here we provide the explicit form of the completely positive dynamical map $\mu_{i}$ ruling the evolution of each optomechanical system. We assume bright input lasers, so that the quantum part of the optomechanical dynamics occurs at the level of the fluctuations of the mirrors' and fields' quadratures around their respective classical mean values. The latter can be easily determined by means of a standard mean-field approach ${ }^{30}$, while the former obey quantum Langevin equations that can be cast compactly as

$$
\partial_{t} \hat{\mathrm{f}}_{\mathrm{i}}=\mathrm{K}_{i} \hat{\mathrm{f}}_{\mathrm{i}}+\mathrm{n}_{\mathrm{i}}, \quad(\mathrm{i}=1,2)
$$

where $\hat{\mathrm{f}}_{i}^{T}=\left(\delta \hat{\mathrm{Q}}_{i}, \delta \hat{P}_{i}, \delta \hat{x}_{i}, \delta \hat{y}_{i}\right)$ is the ordered vector of the fluctuations of the dimensionless quadrature operators $\hat{Q}_{i}=\hat{q}_{i} \sqrt{m_{i} \omega_{m}^{i} / \hbar}$ and $\hat{P}_{i}=\hat{p}_{i} / \sqrt{\hbar m_{i} \omega_{m}^{i}}$ for mechanical mode $i$ and $\delta \hat{x}_{i}=\left(\delta \hat{c}_{i}^{\dagger}+\delta \hat{c}_{i}\right) / \sqrt{2}, \delta \hat{y}_{i}=i\left(\delta \hat{c}_{2}^{\dagger}-\delta \hat{c}_{i}\right) / \sqrt{2}$ for the corresponding cavity fields. Each $4 \times 4$ kernel matrix $\mathrm{K}_{i}$ reads

$$
\mathrm{K}_{i}=\left[\begin{array}{cccc}
0 & \omega_{m}^{i} & 0 & 0 \\
-\omega_{m}^{i} & -\gamma_{m}^{i} & 2 g_{i} \Re\left[c_{s}^{i}\right] & 2 g_{i} \Im\left[c_{s}^{i}\right] \\
-2 g_{i} \Im\left[c_{s}^{i}\right] & 0 & -\kappa_{i} & \Delta_{i} \\
2 g_{i} \Re\left[c_{s}^{i}\right] & 0 & -\Delta_{i} & -\kappa_{i}
\end{array}\right] \quad(i=1,2)
$$

with $g_{i}=\chi_{i} \sqrt{\hbar /\left(2 m_{i} \omega_{m}^{i}\right)}$ the effective coupling rate, $c_{s}^{i}=\varepsilon_{i} /\left(\kappa_{i}+i \Delta_{i}\right)$ the mean amplitude of the $i^{\text {th }}$ cavity field and $\Delta_{i}=\omega_{C}^{i}-\omega_{L}-\frac{h \gamma_{i}^{2}\left|c_{i}^{i}\right|^{2}}{m_{i} \omega_{m}^{2}}$ the cavity-laser detuning modified by each mirror's mean position. The last term in Eq. (2) is the vector of input noise $\mathrm{n}_{j}^{T}(t)=\left(0, \hat{\xi}_{i}(t), \sqrt{2 \kappa_{i}} \delta \hat{x}_{i n}^{i}(t), \sqrt{2 \kappa_{i}} \delta \hat{y}_{i n}^{i}(t)\right)$, where $\hat{\xi}_{i}(t)$ is the zero-mean Langevin force operator accounting for the Brownian motion affecting the mechanical mode $i$. For large mechanical quality factors, $\hat{\xi}_{i}$ is correlated as $\left\langle\hat{\xi}_{i}(t) \hat{\xi}_{i}\left(t^{\prime}\right)\right\rangle=2 \gamma_{m}^{i} k_{B} T_{i} \delta\left(t-t^{\prime}\right) / \hbar \omega_{m}^{i}$, with $k_{B}$ the Boltzmann constant and $T_{i}$ the temperature of the $i^{\text {th }}$ mechanical bath, while $\delta \hat{x}_{i n}^{i}=\left(\delta \hat{c}_{i n}^{i \dagger}+\delta \hat{c}_{i n}^{i}\right) / \sqrt{2}$ and $\delta \hat{y}_{i n}^{i}=i\left(\delta \hat{c}_{i n}^{i \dagger}-\delta \hat{c}_{i n}^{i}\right) / \sqrt{2}$ are the quadratures of the input noise to a cavity. At typical optical frequencies, the latter are delta-correlated as $\left\langle\delta \hat{c}_{i n}^{j}(t) \delta \hat{c}_{i n}^{k \dagger}\left(t^{\prime}\right)\right\rangle=$ $\delta_{j k} \delta\left(t-t^{\prime}\right)$ with $\left\langle\delta \hat{c}_{i n}^{j \dagger}(t) \delta \hat{c}_{i n}^{k}\left(t^{\prime}\right)\right\rangle=\left\langle\delta \hat{c}_{i n}^{j}(t) \delta \hat{c}_{i n}^{k}\left(t^{\prime}\right)\right\rangle=0 \forall j, k$. Eqs. (2) are solved to get the formal expression for the map $\hat{\Phi}$ as

$$
\hat{\mathrm{f}}_{i}(t) \equiv \hat{\Phi}\left[\hat{\mathrm{f}}_{i}(0)\right]=e^{\mathrm{K}_{i} t} \hat{\mathrm{f}}_{i}(0)+\int_{0}^{t} d t^{\prime} e^{\mathrm{K}_{i} t^{\prime}} \hat{\mathrm{n}}_{i}\left(t-t^{\prime}\right) \cdot(4)
$$

Quantum entanglement and discord in two-mode CV states. A general two-mode Gaussian state is fully identified by specifying the elements $v_{i j}=\left\langle\hat{x}_{i} \hat{x}_{j}+\hat{x}_{j} \hat{x}_{i}\right\rangle / 2$ of the covariance matrix $v$, where we have introduced $\hat{x}=\left(\hat{q}_{1}, \hat{p}_{1}, \hat{q}_{2}, \hat{p}_{2}\right)$ as the vector of the quadrature operators $\hat{q}_{i}$ and $\hat{p}_{i}(i=1,2)$ of the two-mode system. Through Eq. (4), the time behavior of the four-mode covariance matrix $v_{\mathrm{f}}=\left\langle\hat{\mathrm{f}}_{\mathrm{i}} \hat{\mathrm{f}}_{j}+\hat{\mathrm{f}}_{j} \hat{\mathrm{f}}_{i}\right\rangle / 2$ of our system can be determined explicitly, thus completely solving the dynamical problem. Any covariance matrix can be written as

$$
v=\left[\begin{array}{cc}
\alpha_{1} & \gamma \\
\gamma^{T} & \alpha_{2}
\end{array}\right]
$$

where $\alpha_{1}\left(\alpha_{2}\right)$ and $\gamma$ are $2 \times 2$ matrices accounting for the local variances of mode 1 (2) and the inter-mode correlations. Entanglement can thus be quantified by means of the logarithmic negativity ${ }^{31,32} E=\max \left[0,-\ln 2\left(v_{-}\right)\right]$with $v_{-}$the smallest element of the symplectic spectrum of the partially transposed covariance matrix $v^{P}=\mathrm{P} v \mathrm{P}$ (with $\mathrm{P}=\hat{\mathrm{I} l} \oplus \sigma_{z}$ ). The symplectic spectrum of a matrix $\mu$ is given by the eigenvalues of $\left|i\left(\oplus_{i=1}^{2} i \sigma_{y}^{i}\right) \mu\right|\left(\sigma_{i}\right.$ is the $i=x, y, z$ Pauli matrix $)$. Gaussian discord, on the other hand, is calculated as ${ }^{13,14}$

$$
D=f\left(\sqrt{A_{2}}\right)-f\left(\mu_{-}\right)-f\left(\mu_{+}\right)+\inf _{v_{0}} f(\sqrt{\operatorname{det} \varepsilon}) .
$$

Here, $f(x)=\left(\frac{x+1}{2}\right) \log \left[\frac{x+1}{2}\right]-\left(\frac{x-1}{2}\right) \log \left[\frac{x-1}{2}\right], A_{2}=\operatorname{det} \alpha_{2}, \mu_{ \pm}$are the symplectic eigenvalues of $v, \varepsilon=\alpha_{1}-\gamma\left(\alpha_{2}+v_{0}\right)^{-1} \gamma^{T}$ is the Schur complement of $\alpha_{1}$, and $v_{0}$ is the covariance matrix of a general single-mode rotated squeezed state, over whose parameters $\inf _{v_{0}} f(\sqrt{\operatorname{det} \varepsilon})$ should be evaluated, which can be done analytically for general two-mode covariance matrices of Gaussian states ${ }^{13,14}$.

1. Horodecki, R., Horodecki, P., Horodecki, M. \& Horodecki, K. Quantum entanglement. Rev. Mod. Phys. 81, 865-942 (2009).

2. Olliver, H. \& Zurek, W. H. Quantum discord: a measure of the quantumness of correlations. Phys. Rev. Lett. 88, 017901-017904 (2001).

3. Henderson, L. \& Vedral, V. Classical, quantum and total correlations. J. Phys. A 34, 6899-7905 (2001).

4. Oppenheim, J., Horodecki, M., Horodecki, P. \& Horodocki, R. Thermodynamical approach to quantifying quantum correlations. Phys. Rev. Lett. 89, $180402-$ 180405 (2002).

5. Groisman, B., Popescu, S. \& Winter, A. Quantum, classical and total amount of correlations in a quantum state. Phys. Rev. A 72, 032317-032327 (2005). 
6. Luo, S. Using measurement-induced disturbance to characterize correlations as classical or quantum. Phys. Rev. A 77, 022301-022305 (2008).

7. Modi, K., Paterek, T., Son, W., Vedral, V. \& Williamson, M. Unified view of quantum and classical correlations. Phys. Rev. Lett. 104, 080501-080503 (2010).

8. Datta, A., Shaji, A. \& Caves, C. M. Quantum discord and the power of one qubit. Phys. Rev. Lett. 100, 050502-050505 (2008).

9. Madhok, V. \& Datta, A. Interpreting quantum discord through quantum state merging. Phys. Rev. A 83, 032323-032326 (2011).

10. Cavalcanti, D., et al., Operational interpretations of quantum discord. Phys. Rev. A 83, 032324-032328 (2011).

11. Streltsov, A., Kampermann, H., \& Bruss, D. Linking quantum discord to entanglement in a measurement. Phys. Rev. Lett. 106, 160401-160403 (2011).

12. Piani, M. et al., All nonclassical correlations can be activated into distillable entanglement. Phys. Rev. Lett. 106, 220403-220406 (2011).

13. Giorda, P. \& Paris, M. G. A. Gaussian quantum discord. Phys. Rev. Lett. 105 020503-020506 (2010).

14. Adesso, G. \& Datta, A. Quantum versus classical correlations in gaussian states. Phys. Rev. Lett. 105, 030501 (2010).

15. Aspelmeyer, M., Gröblacher, S., Hammerer, K. \& Kiesel, N. Quantum optomechanics-throwing a glance. J. Opt. Soc. Am. B 27, A189- A197 (2010).

16. Marquardt, F. \& Girvin, S. M. Optomechanics. Physics 2, 40 (2009);

17. Kippenberg, T. J., \& Vahala, K. J. Cavity opto-mechanics. Optics Express 15, 17172-17205 (2007).

18. Rocheleau, T., et al., Preparation and detection of a mechanical resonator near the ground state of motion. Nature 463, 72-75 (2010).

19. Teufel, J. D., et al., Sideband cooling of micromechanical motion to the quantum ground state. Nature 475, 359-363 (2011).

20. Gröblacher, S., Hammerer, K., Vanner, M. R., \& Aspelmeyer, M. Observation of strong coupling between a micromechanical resonator and an optical cavity field. Nature 460, 724-727 (2009).

21. Connell, A. D. O', et al., Quantum ground state and single-phonon control of a mechanical resonator. Nature 464, 697-703 (2010).

22. Vitali, D., et al., Optomechanical Entanglement between a Movable Mirror and a Cavity Field. Phys. Rev. Lett. 98, $030405-030408$ (2007).

23. Mancini, S., Giovannetti, V., Vitali, D. \& Tombesi, P. Entangling Macroscopic Oscillators Exploiting Radiation Pressure. Phys. Rev. Lett. 88, 120401-120403 (2002).

24. Pinard, M., et al., Entangling movable mirrors in a double-cavity system. Europhys. Lett. 72, 747-753 (2005).
25. Pirandola, S., Vitali, D., Tombesi, P. \& Lloyd, S. Macroscopic entanglement by entanglement swapping. Phys. Rev. Lett. 97, 150403- 150406 (2006).

26. Vitali, D., Mancini, S. \& Tombesi, P. Stationary entanglement between two movable mirrors in a classically driven FabryPerot cavity. J. Phys. A 40, 8055-8068 (2007).

27. Paternostro, M., et al., Creating and Probing Multipartite Macroscopic Entanglement with Light. Phys. Rev. Lett. 99, 250401-250404 (2007).

28. Hartmann, M. J. \& Plenio, M. B. Steady State Entanglement in the Mechanical Vibrations of Two Dielectric Membranes. Phys. Rev. Lett. 101, 200503-200506 (2008).

29. Mazzola, L. \& Paternostro, M. Distributing fully optomechanical quantum correlations. Phys. Rev. A 83, 062335-062339 (2011).

30. Walls, D. F. \& Milburn, G. J. Quantum Optics (Springer-Verlag, Heidelberg, 2008).

31. Zyczkowski, K., Horodecki, P., Sanpera, A. \& Lewenstein, M. Volume of the set of separable states. Phys. Rev. A 58, 883-892 (1998).

32. Vidal, G. \& Werner, R. F., Computable measure of entanglement. Phys. Rev. A 65, 032314-032324 (2002).

\section{Acknowledgments}

We thank M. Piani and G. Adesso for discussions. We acknowledge the Magnus Ehrnrooth Foundation and the UK EPSRC (EP/G004759/1) for financial support.

\section{Author Contributions}

Both the authors have made a significant contribution to the concept, execution and interpretation of the presented work.

\section{Additional information}

Competing financial interests: The Authors declare no competing financial interests.

PACS numbers: 42.50.Pq, 03.67.Mn, 03.65.Yz

License: This work is licensed under a Creative Commons

Attribution-NonCommercial-NoDerivative Works 3.0 Unported License. To view a copy of this license, visit http://creativecommons.org/licenses/by-nc-nd/3.0/

How to cite this article: Mazzola, L. \& Paternostro, M. Activating optomechanical entanglement. Sci. Rep. 1, 199; DOI:10.1038/srep00199 (2011). 